\title{
MRI findings in proven Mycobacterium tuberculosis (TB) spondylitis
}

\section{J Kotzé, MB ChB \\ L J Erasmus, $M B C h B$}

Department of Diagnostic Radiology, University of the Free State, Bloemfontein

\section{Abstract}

The incidence of skeletal TB is increasing. A better understanding of the MRI features of proven TB spondylitis in our setting is needed.

Materials and methods. Histologically proven cases of TB spondylitis, with MR imaging performed at Universitas Hospital on a $1.5 \mathrm{~T}$ scanner, were reviewed.

Results. Typical findings of vertebral column involvement were seen in all patients, namely multiple levels affected and paravertebral abscesses.

The thoracic spine was involved more than the lumbar spine. In nearly all cases intervertebral disc involvement was noted. Posterior longitudinal ligaments were intact in all but 1 patient, even though there was some elevation in a number of patients. Abscess walls were also found to be thick instead of thin as expected.

\section{Introduction}

There is a world-wide increased incidence of tuberculosis (TB), with the HIV pandemic contributing significantly to this trend. ${ }^{1,2}$ The most common extrapulmonary location of TB is the spine; in over 50\% of the cases of bone and joint involvement. ${ }^{3}$ Because MRI facilities are more readily available, imaging of suspected spinal TB using this modality has increased. MRI is now the preferred imaging modality for patients with suspected spinal TB. ${ }^{4,5}$ We wanted to evaluate the imaging features of histologically proven cases in our setting.

MRI features that suggest TB are:

- soft-tissue masses/abscesses

- involvement of multiple vertebral segments of the spine

- absence of reactive sclerosis.

\section{Materials and methods}

We identified all patients who had vertebral biopsies for suspected TB spondylitis, from July 2002 until November 2005. Of these, we selected all patients who had histology results confirming TB spondylitis, and a recent MRI of the affected vertebrae. Twenty-three patients conformed to the above-mentioned criteria, (11 male and 12 female). Patients' age ranged from 17 to 75 years, with an average age of 36 years.

All MR studies were performed at Universitas Hospital, on a GE Signa 1.5Tesla MR, with all patients imaged with $\mathrm{T} 1$ and $\mathrm{T} 2$ multiplanar spin echo sequences.

Additional STIR images were acquired for 10 of these patients, while gadolinium was administered in 16 cases.

Images were assessed by an experienced radiologist. Pre-prepared recording sheets were used. The histology results were known to the assessor.

Limitations of the study include examiner subjectivity regarding some findings, especially abscess wall thickness, and the fact that no follow-up imaging was performed after treatment.

\section{Abnormalities}

The following abnormalities were assessed:

1. Vertebral involvement

- level(s) affected

- number of vertebrae involved

- complete destruction

- partial destruction (including anterior, posterior and diffuse vertebral body involvement)

- signal intensities in partially affected vertebrae

- iso-intense, hyper-intense or hypo-intense compared with normal vertebrae

2. Disc involvement

- Destruction

- Partial destruction (including height loss and fragmentation)

- Complete destruction

- Signal changes in partially destructed discs

3. Paravertebral abscess/soft-tissue mass formation

- Anterior location/ posterior location with intraspinal extension

- Thin or thick walled

- Septae present

4. Longitudinal ligament involvement

- Elevation

- Destruction

- Anterior/posterior location

Table I. Level of epicentre of vertebral involvement

\begin{tabular}{lll}
\hline & $\mathbf{N = 2 3}$ & $\%$ \\
\hline High thoracic (T1-4) & 4 & 17 \\
Mid thoracic (T5-8) & 6 & 26 \\
Lower thoracic (T9-12) & 6 & 26 \\
L1 & 0 & 0 \\
L2 & 2 & 9 \\
L3 & 2 & 9 \\
L4 & 3 & 13 \\
L5 & 0 & 0
\end{tabular}




\section{ORIGINAL ARTICLE}

Table II. Distribution of partial destruction

\begin{tabular}{llll}
\hline Part of vertebral body & Anterior and posterior & Only anterior & Only posterior \\
\hline Thoracic & 13 of $16(81 \%)$ & 2 of 16 & 1 of 16 \\
Lumbar & 7 of $7(100 \%)$ & 0 & 0 \\
\hline
\end{tabular}

\begin{tabular}{|c|c|c|c|c|c|c|c|}
\hline \multicolumn{8}{|c|}{ Table III. Signal intensities in partially affected vertebrae } \\
\hline & Iso-intense & $\%$ & High signal & $\%$ & Low signal & $\%$ & Total \\
\hline $\mathrm{T} 1$ & 6 of 23 & 26 & 0 of 23 & 0 & 17 of 23 & 74 & 23 \\
\hline $\mathrm{T} 2$ & 3 of 23 & 13 & 17 of 23 & 74 & 3 of 23 & 13 & 23 \\
\hline Post Gd & 3 of 16 & 19 & 13 of 16 & 81 & 0 of 23 & 0 & 16 \\
\hline STIR & 1 of 10 & 10 & 9 of 10 & 90 & 0 & 0 & 10 \\
\hline
\end{tabular}

\section{Results}

\section{Vertebral involvement}

There was complete destruction of at least one vertebral body in 14 patients (69\%) (11 of $16(69 \%)$ patients with thoracic and 3 of 7 (43\%) with lumbar involvement).

Tables I-III show the levels of vertebral involvement, distribution of partial destruction and signal intensities in partially affected vertebrae.

\section{Disc involvement}

Tables IV and V show the extent of disc involvement and signal intensities in partially affected discs.

\begin{tabular}{lll} 
Table IV. Extent of disc involvement & & \\
\hline & N=23 & $\%$ \\
\hline No involvement & 1 & 4 \\
Complete destruction & 8 & 30 \\
Partial destruction only & 7 & 35 \\
Partially and completely destructed discs & 7 & 30
\end{tabular}

\section{Paravertebral abscess formation}

Paravertebral abscess formation is shown in Table VI.

\section{Longitudinal ligament involvement}

Table VII lists the longitudinal ligament involvement.

\section{Discussion}

Tuberculous spondylitis/spondylodiscitis is caused by the Mycobacterium tuberculosis bacillus. The features of the disease were first described by Percival Pott. The disease has potentially serious morbidity with severe neurological impairment and disfiguring deformity.

Also known as Pott's disease, spinal infection follows haematogenous seeding from a distant source, and an extraspinal source of infection should be considered. The basic lesion is a combination of osteomyelitis and arthritis. Typically, more than one vertebra is involved. The area usually affected is the anterior aspect of the vertebral body adjacent to the subchondral plate. TB may spread from that area to adjacent intervertebral discs. In adults, disc disease is secondary to the spread of infection from the vertebral body. In children, because the disk is vascularised, it can be a primary site.

Vertebral collapse and kyphosis follows progressive bone destruction. Spinal canal luminal diameter narrowing is due to abscess formation, granulation tissue, or direct dural invasion. Consequently there is

\begin{tabular}{|llllllll|}
\hline \multicolumn{7}{|c|}{ Table V. Signal intensities in partially affected discs } \\
\hline T1 & Iso-intense & $\%$ & High signal & $\%$ & Low signal & $\%$ & Total \\
T2 & 11 of 14 & 79 & 0 of 14 & 0 & 3 of 14 & 21 & 14 \\
Post Gd & 0 of 14 & 0 & 8 of 14 & 57 & 6 of 14 & 43 & 14 \\
STIR & 0 of 10 & 0 & 3 of 10 & 30 & 7 of 10 & 70 & 10 \\
& 0 of 5 & 0 & 2 of 5 & 40 & 3 of 5 & 60 & 5 \\
\hline
\end{tabular}




\section{ORIGINAL ARTICLE}

\begin{tabular}{|lll|}
\hline \multicolumn{3}{|c|}{ Table VI. Paravetebral abscess formation } \\
\hline & $N=\mathbf{2 3}$ & $\%$ \\
\hline Only anterior & 1 & 4 \\
Only posterior (with spinal extension) & 1 & 4 \\
Anterior and posterior (with spinal extension) & 21 & 92 \\
Thin wall & 9 & 39 \\
Thick, irregular wall & 14 & 61 \\
Septae present & 18 & 78 \\
\hline
\end{tabular}

\begin{tabular}{|lll|}
\hline \multicolumn{3}{|c|}{ Table VII. Longitudinal ligament involvement } \\
\hline & $N=\mathbf{2 3}$ & $\%$ \\
\hline None & 0 & 0 \\
$\begin{array}{l}\text { Elevation anterior and posterior, without } \\
\text { destruction }\end{array}$ & 12 & 52 \\
$\begin{array}{l}\text { Elevation and destruction anterior with only } \\
\text { elevation posterior }\end{array}$ & 10 & 43 \\
$\begin{array}{l}\text { Elevation and destruction posterior, without } \\
\text { anterior involvement }\end{array}$ & 1 & 4 \\
\hline
\end{tabular}

spinal cord compression and thus neurological deficits. Kyphotic deformity may follow collapse of the anterior vertebral bodies. Paraspinal abscesses in the lumbar region gravitate along the sheath of the psoas to the femoral region and erosion through to the skin may follow.

In our study the epicentre of involvement was at the thoracic level in 16 of 23 patients (69\%), with the epicentre in the remainder (31\%) at the lumbar level.

This is contrary to the findings of Sinan et al. ${ }^{6}$ where involvement was greater in the lumbar spine. With thoracic involvement, the distribution at different levels (high, mid and lower) was fairly equal.

None of the patients had L1 or L5 as the epicentre of involvement.

There was complete destruction of at least one vertebral body in 14 patients (69\%). Partially affected vertebrae showed destructive changes in both the anterior and posterior parts of the vertebral body in $87 \%$ (20 of 23). Signal intensities in partially affected vertebrae were predominantly hypo-intense on T1 (74\%), hyper-intense on T2 (74\%), hyperintense after gadolinium administration (81\%), and hyper-intense on STIR sequences (Figs 1 - 3).

All patients, with the exception of one who had no disc involvement, had varying degrees of complete and partial disc destruction at different levels.

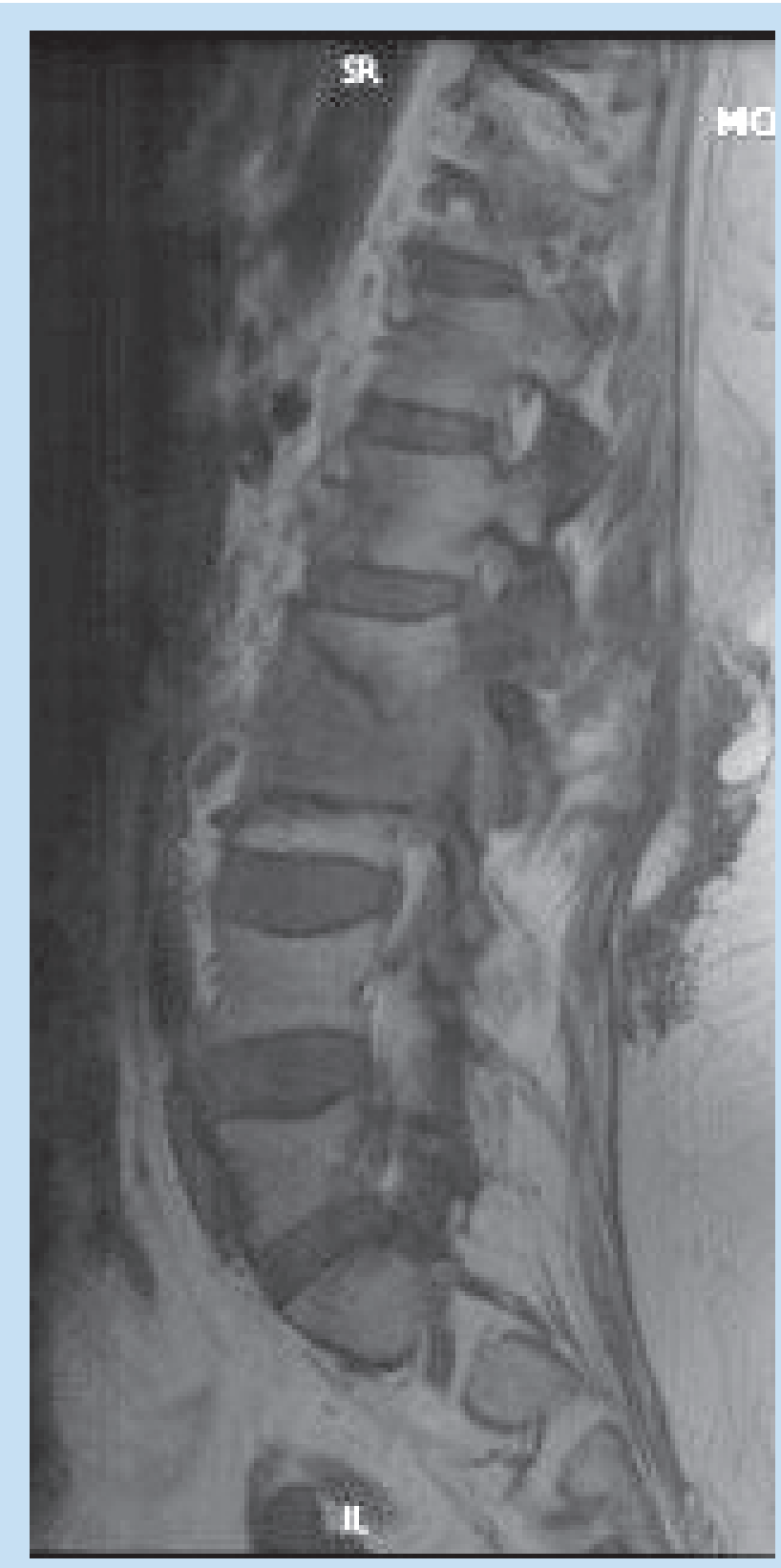

Fig 1. T1 sagittal image. Partial destruction of the anterior and posterior vertebral bodies of $L 2$ and $L 3$.

All 23 patients showed paravertebral abscess formation, extending between 2 and 6 vertebral levels. In the majority of patients ( 21 of $23,91 \%)$ there was extension of the abscess adjacent to the anterior and posterior aspects of the vertebral bodies, while 61\% (14 of 23) of the abscesses had a thick irregular wall, with a thin smooth wall in the remainder. Septae were present in 18 of 23 abscesses (78\%). Sclerosis was 


\section{ORIGINAL ARTICLE}

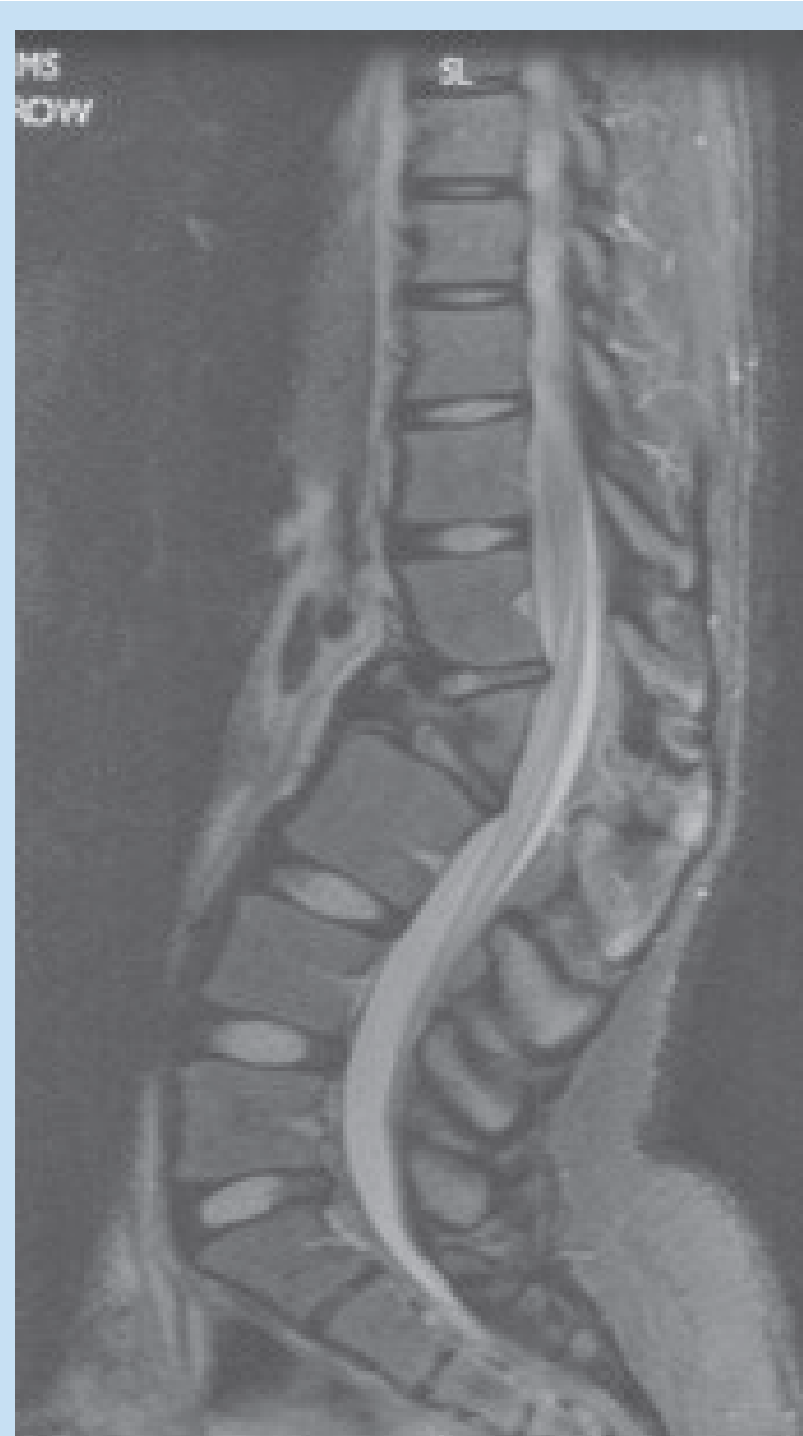

Fig 2. T2 sagittal image. Predominantly anterior vertebral body destruction of L2, with gibbus formation.

seen in $13 \%$ (3 of 23) of affected vertebrae (Fig. 4).

In all 23 patients there was longitudinal ligament involvement, with:

- Elevation anterior and elevation posterior in 12 (52\%)

- Elevation and destruction anterior, with only elevation posterior in $10(44 \%)$

- Elevation and destruction posterior without anterior involvement in $1(4 \%)$.

Fig. 5 shows elevation with an intact posterior longitudinal ligament.



Fig 3a. T1 sagittal image. Low signal in affected thoracic vertebrae. 


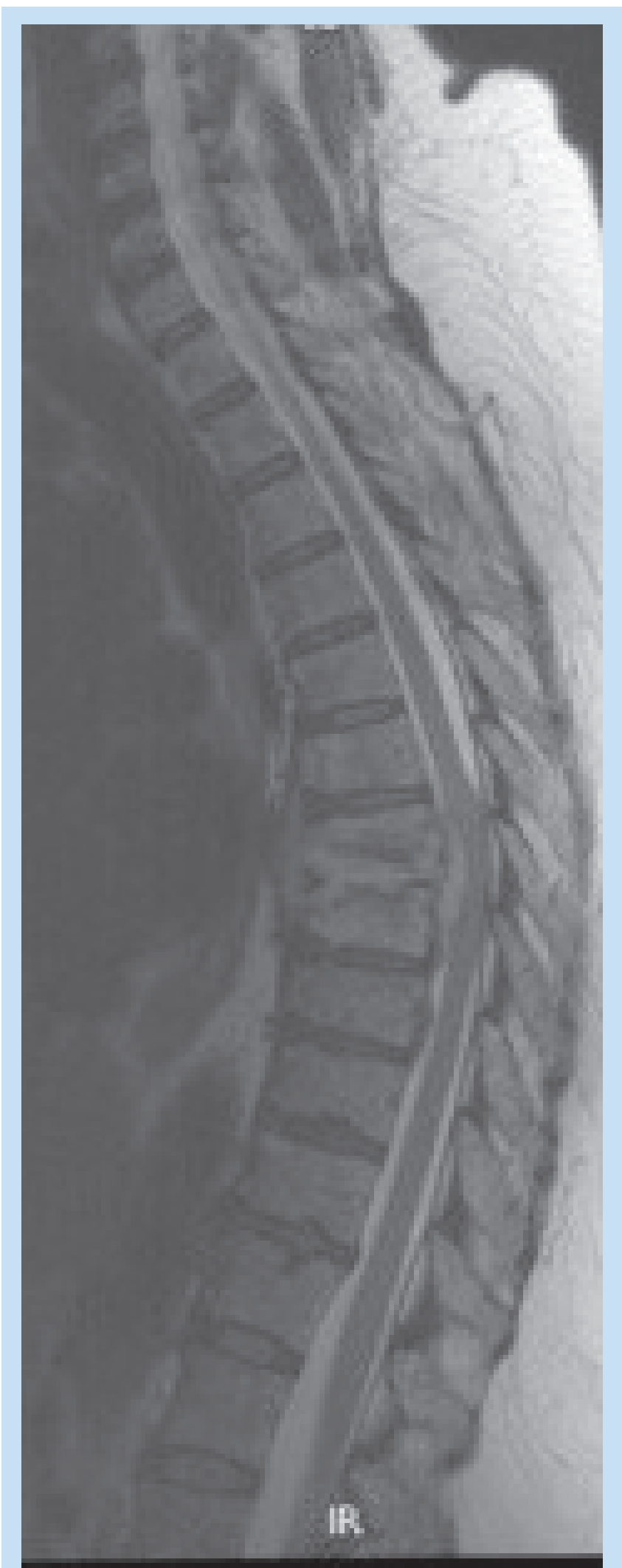

Fig 3b. T2 sagittal image. High signal in affected vertebrae.

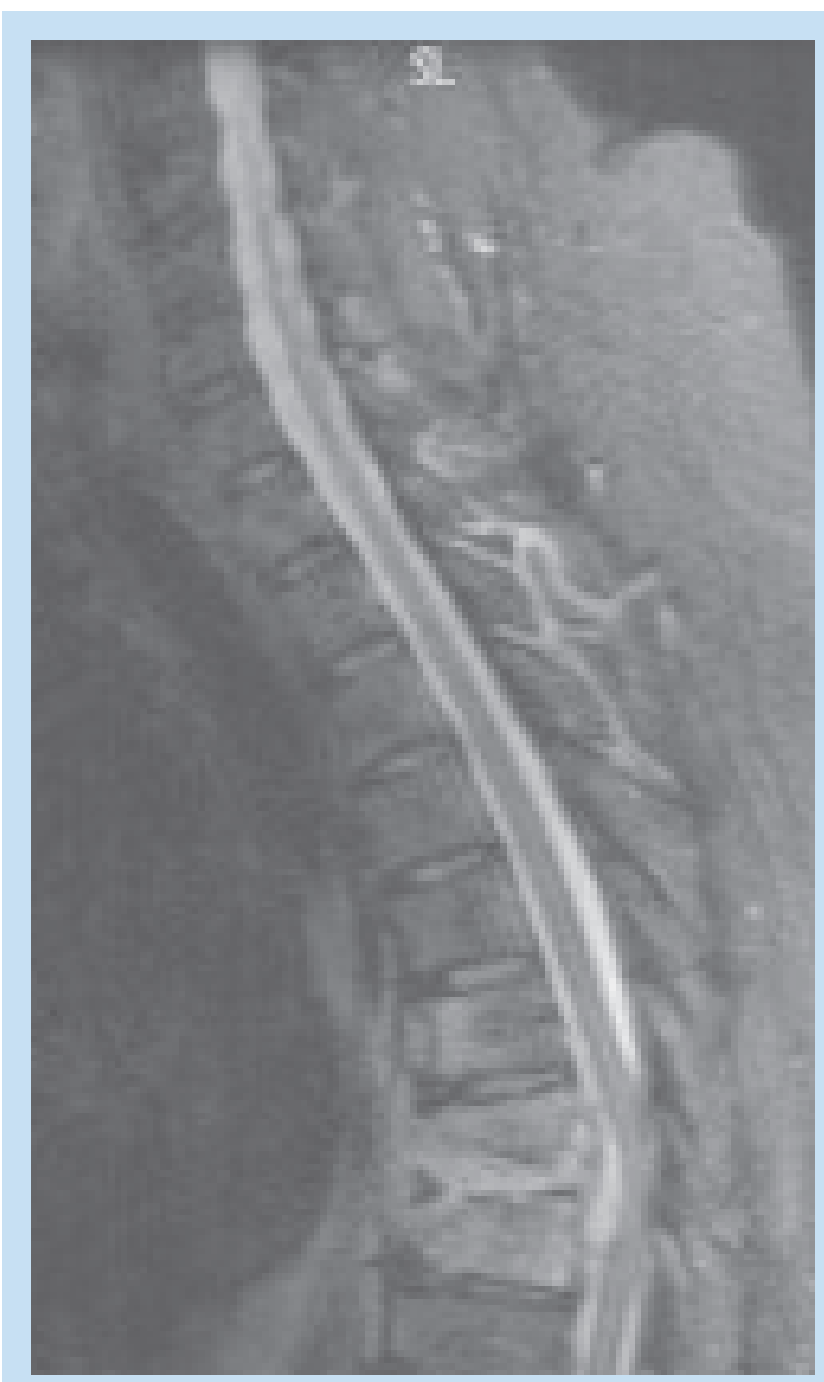

Fig 3c. Sagittal STIR image. High signal in affected vertebrae.

\section{Conclusion}

This study confirmed the findings of previous studies, namely:

- Paravertebral abscess formation involving multiple levels ${ }^{7-9}$

- Subligamentous spread to multiple levels ${ }^{6}$

- Hyper-intensity of affected vertebrae on T2 images ${ }^{6}$

- Hypo-intensity of affected vertebrae on T1 images $^{6}$

We found that abscess walls were thickened in about $60 \%$ in contrast to previously reported thin, smooth walls. ${ }^{6}$

In our study all but one patient had at least partial disc destruction.

The posterior longitudinal ligaments were elevated in all patients, but only destroyed in one. Anterior ligaments were elevated in 22 patients, with associated destruction in $10(43 \%)$. These findings have not previously been specifically reported.

Further studies that focus on the pattern of ligament involvement and the abscess walls would be of value. Objective measurement of the abscess walls would obviate examiner subjectivity. 


\section{ORIGINAL ARTICLE}

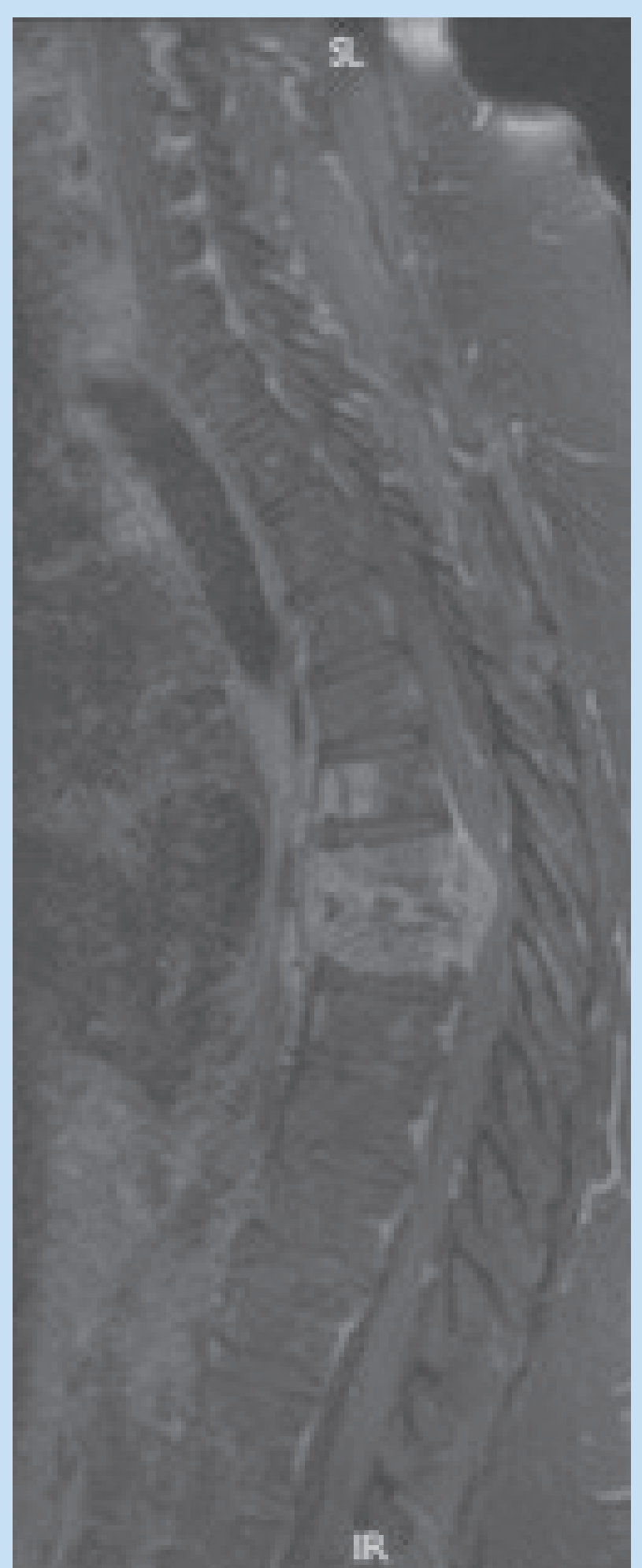

Fig 3d. T1 postcontrast sagittal image. Enhancement of the affected vertebrae.



Fig 4a. T2 axial image.

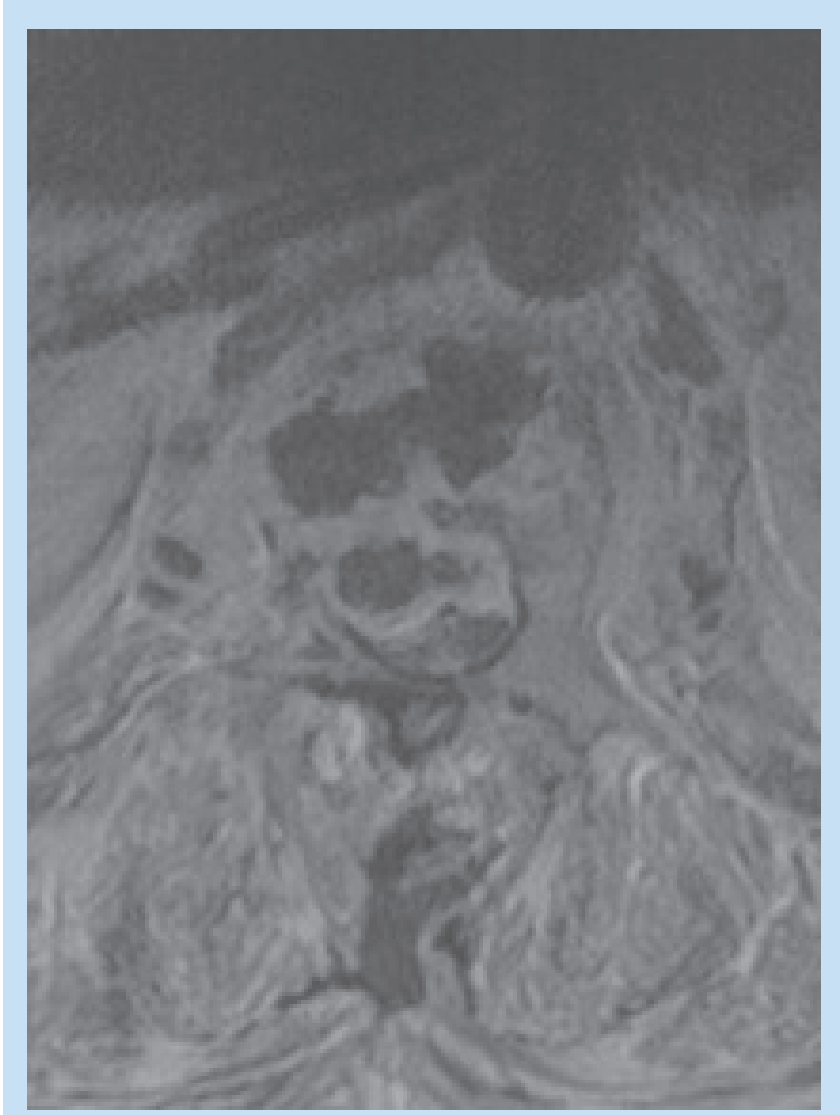

Fig 4b. T1 postcontrast axial image. Demonstrating thick irregular paravertebral abscess walls. 


\section{ORIGINAL ARTICLE}

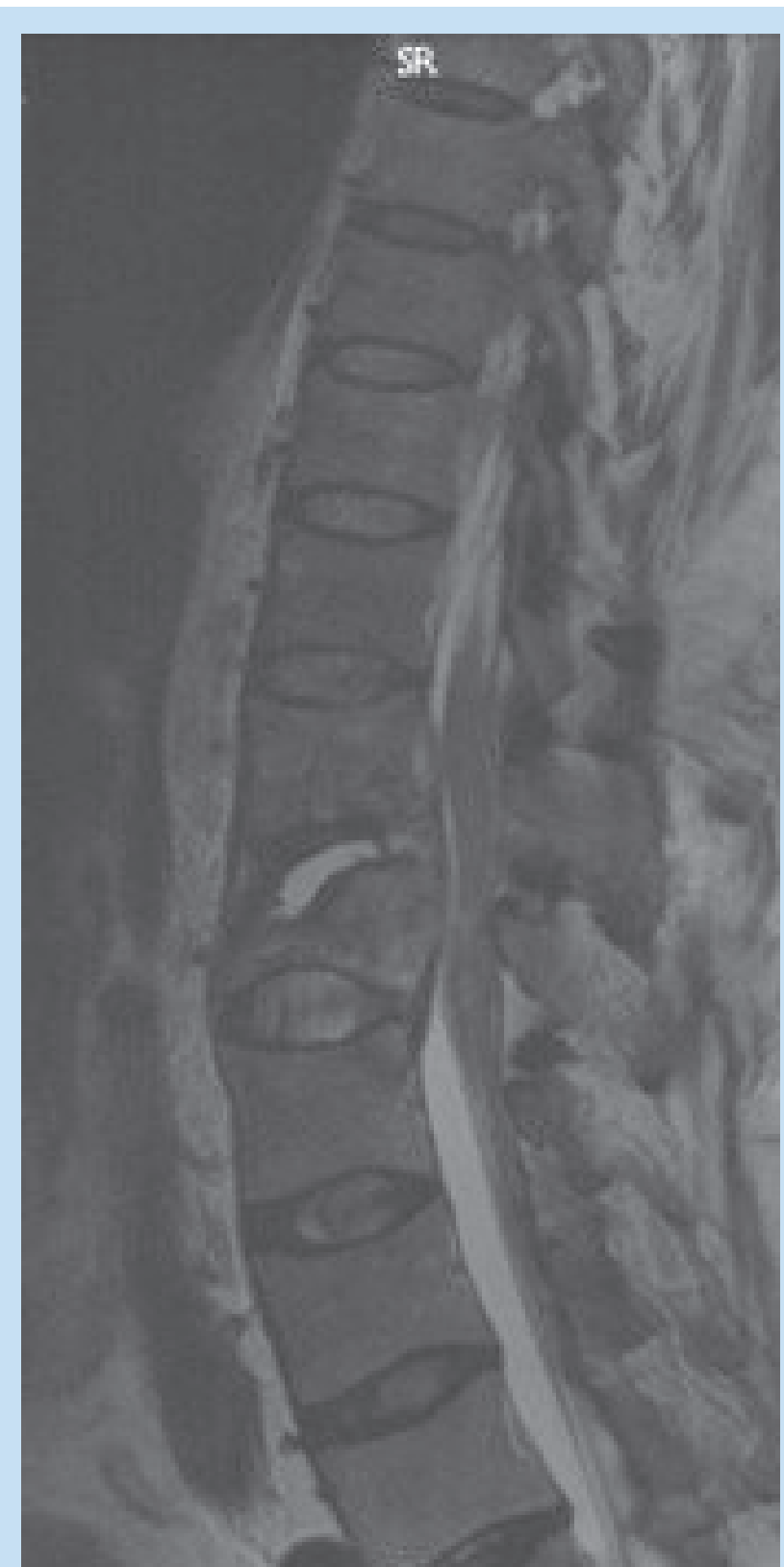

Fig 5. T2 sagittal image. Elevated, but intact posterior longitudinal ligament.
REFERENCES

1. Restrepo CS, Lemos DF, Gordillo H, et al. Imaging findings in musculoskeletal complications of AIDS Radiographics 2004; 24:1029-1049.

2. Engin G, Acuna B, Acuna G, Tunaci M. Imaging of extrapulmonary tuberculosis. Radiographics 2000; 20:471-488.

3. Tuberculous spodylitis; Wheeless Textbook of Orthopaedics. Duke University Medical Center, http://www. wheelessonline.com online book.

4. Kotevoglu N, Tasbasi I. Diagnosing tuberculous spondylitis: patients with back pain referred to a rheumatology outpatient department. Rheumatol Int 2004; 24: 9-13.

5. Hidalgo JA, Alangaden G. http://www.emedicine.com/med/topic 1902.htm eMedicine online article- Pott disease July 2005.

6. Sinan T, Al-Khawari H, Ismail M, Ben-Nakhi A, Sheikh M. Spinal tuberculosis: CT and MRI features. Ann Saudi Med 2004; 24(6): 437-441.

7. De Vuyst D, Vanhoenacker F, Gielen J, Bernaerts A, De Schepper AM. Imaging features of musculoskeletal tuberculosis. Eur Radiol 2003; 13: 1809-1819.

8. Khoo LT, Mikawa K, Fessler R. A surgical revisitation of Pott distemper of the spine. The Spine Journal 2003; 3: 130-145.

9. NaYoung Jung, Jee WH, Ha KY, Park CK, Byun JY. Discrimination of tuberculous spondylitis from pyogenic spondylitis on MRI. AJR 2004; 182: 1405-1410. 